\title{
Biofilm Formation by Pseudallescheria/Scedosporium Species: A Comparative Study
}

\author{
Rodrigo Rollin-Pinheiro ${ }^{1+}$, Jardel V. de Meirelles ${ }^{1 \dagger}$, Taissa V. M. Vila ${ }^{2}$, \\ Beatriz B. Fonseca ${ }^{2}$, Vinicius Alves ${ }^{3}$, Susana Frases ${ }^{3}$, Sonia Rozental ${ }^{2}$ and \\ Eliana Barreto-Bergter ${ }^{1 *}$
}

\begin{abstract}
'Laboratório de Química Biológica de Microrganismos, Departamento de Microbiologia Geral, Instituto de Microbiologia Paulo de Goes, Universidade Federal do Rio de Janeiro, Rio de Janeiro, Brazil, ${ }^{2}$ Laboratório de Biologia Celular de Fungos, Departamento de Parasitologia e Biologia Celular, Instituto de Biofísica, Universidade Federal do Rio de Janeiro, Rio de Janeiro, Brazil, ${ }^{3}$ Laboratório de Ultraestrutura Celular Hertha Meyer, Departamento de Parasitologia e Biologia Celular, Instituto de Biofísica, Universidade Federal do Rio de Janeiro, Rio de Janeiro, Brazil
\end{abstract}

\section{OPEN ACCESS}

Edited by:

Helio K. Takahashi,

Federal University of São Paulo, Brazil

Reviewed by: Jean-Philippe Bouchara,

University of Angers, France Luciana Lopes Guimaraes, Universidade Santa Cecilia, Brazil

${ }^{*}$ Correspondence:

Eliana Barreto-Bergter eliana.bergter@micro.ufri.br

${ }^{\dagger}$ These authors have contributed equally to this work.

Specialty section: This article was submitted to Fungi and Their Interactions,

a section of the journal

Frontiers in Microbiology

Received: 29 June 2017 Accepted: 02 August 2017 Published: 18 August 2017

Citation: Rollin-Pinheiro $R$, de Meirelles $J$ Vila TVM, Fonseca BB, Alves $V$,

Frases S, Rozental S and Barreto-Bergter E (2017) Biofilm Formation by Pseudallescheria/Scedosporium Species: A Comparative Study.

Front. Microbiol. 8:1568. doi: 10.3389/fmicb.2017.01568
Pseudallescheria/Scedosporium species are medically important fungi that are present in soil and human impacted areas and capable of causing a wide spectrum of diseases in humans. Although little is known about their pathogenesis, their growth process and infection routes are very similar to those of Aspergillus species, which grow as biofilms in invasive infections. All nine strains tested here displayed the ability to grow as biofilms in vitro and to produce a dense network of interconnected hyphae on both polystyrene and the surfaces of central venous catheters, but with different characteristics. Scedosporium boydii and S. aurantiacum clinical isolates were able to form biofilms faster than the corresponding environmental strains, as evidenced in kinetic assays for $S$. boydii and CLSM for $S$. aurantiacum. Biofilms formed by Pseudallescheria/Scedosporium species had significantly higher resistance to the class of antifungal azole than was observed in planktonic cells, indicating a protective role for this structure. In addition, the clinical $S$. aurantiacum isolate that formed the most robust biofilms was also more virulent in a larvae Galleria mellonella infection model, suggesting that the ability to form biofilms enhances virulence in Pseudallescheria/Scedosporium species.

Keywords: fungal biofilms, Scedosporium, Pseudallescheria, virulence, antifungal susceptibility

\section{INTRODUCTION}

Pseudallescheria/Scedosporium species are a group of medically important fungi associated with a wide spectrum of infections in both immunocompromised and immunocompetent patients (Cortez et al., 2008). These fungi have a worldwide distribution in soil and human impacted areas, and their disease spectrum ranges from localized skin infections, such as mycetoma, to life-threatening invasive infections, including pulmonary pseudallescheriosis/scedosporiosis, which can potentially disseminate to the central nervous system (Summerbell et al., 1989; Tadros et al., 1998; Guarro et al., 2006; Rougeron et al., 2015). 
Pseudallescheriosis/scedosporiosis are opportunistic infections that are usually associated with other pathologies, such as cancer, HIV infection, cystic fibrosis and near drowning (Lamaris et al., 2006; Tammer et al., 2011; Zouhair et al., 2013). Scedosporium (Pseudallescheria) boydii, S. apiospermum, and S. aurantiacum are considered clinically relevant species, while others, such as Pseudallescheria ellipsoidea and $P$. angusta, are typically classified as environmental species (Guarro et al., 2006; Cortez et al., 2008; Bouchara et al., 2009). Although there has been an alarming increase in Pseudallescheria/Scedosporium infections in recent years, little is known about the pathogenesis of these fungi (Al Refai et al., 2002; Lamaris et al., 2006; Wilson and Kennedy, 2013).

Biofilms are complex organized communities that are composed of microbial cells surrounded by a self-secreted extracellular polymeric matrix (Davies, 2003; Kaur and Singh, 2014). Biofilm cells are phenotypically different from their planktonic counterparts and, from a clinical point of view, the most relevant difference is an increased resistance to antimicrobials. The presence of a polymeric extracellular matrix has been shown to confer protection against host immune cells and to impair antifungal penetration (Davies, 2003). Aspergillus fumigatus and Candida albicans are the most extensively studied pathogenic fungi that cause biofilm-associated invasive fungal diseases (Sherry et al., 2014; Fan et al., 2015). C. albicans can adhere to medical devices and form biofilms, and these capabilities have commonly been associated with bloodstream infections (Kojic and Darouiche, 2004). In addition, Candida cells that disperse from biofilm structures seem to be more virulent than planktonic cells in animal models (Uppuluri et al., 2010). A. fumigatus is able to form biofilms in patients who present with an aspergilloma and invasive aspergillosis (Muller et al., 2011). These infections are established when A. fumigatus conidia germinate into mycelia embedded in an extracellular matrix, thereby forming a complex biofilm structure inside the host (Filler and Sheppard, 2006; Kaur and Singh, 2014). Once formed, an A. fumigatus biofilm lowers in vitro and in vivo susceptibility to commercially available antifungal drugs (Seidler et al., 2008; Kaur and Singh, 2014).

Fungi in Pseudallescheria/Scedosporium species present a pathogenesis very similar to that of Aspergillus species, in which the germination process is crucial for tissue invasion (Bouchara et al., 2009). Nevertheless, the mechanisms underlying the pathogenesis and virulence of Pseudallescheria/Scedosporium remain unclear. Recently, it was demonstrated that S. apiospermum, S. aurantiacum, S. minutisporum, and Lomentospora prolificans can grow as a biofilm on both polystyrene and tissue culture surfaces (Mello et al., 2016). However, no information is available regarding the correlation between the formation of such biofilms and pathogenicity. Hence, in this study, we aimed to compare the ability of environmental and clinical strains of Pseudallescheria/Scedosporium species to form biofilms in vitro and to correlate these properties with their in vivo pathogenicity in a larvae Galleria mellonella infection model. Additionally, we sought to evaluate the susceptibility of those biofilms to a variety of antifungal drugs belonging to the classes of azoles and echinocandins.

\section{MATERIALS AND METHODS}

\section{Strains and Growth Conditions}

The following strains were used in this study: S. (Pseudallescheria) boydii CBS 120157, S. (Pseudallescheria) boydii CBS 117410, S. (Pseudallescheria) boydii CBS 117432, P. ellipsoidea CBS 301.79, P. angusta CBS 254.72, S. aurantiacum CBS 136910, S. aurantiacum CBS 136046, S. aurantiacum CBS 136047, and S. aurantiacum CBS 136049. They were generously provided by Sybren de Hoog from the Westerdijk Fungal Biodiversity Institute, Utrecht, The Netherlands. A list of all nine strains and where they were isolated is shown in Table 1. All strains were maintained in modified Sabouraud media $(0.5 \%$ yeast extract, $1 \%$ peptone, and $2 \%$ glucose). To obtain conidia, the cells were grown on Sabouraud-agar plates at room temperature, and after 7 days, the surface growth was scraped off using sterile PBS, and the collected conidia were filtered and washed twice with sterile PBS.

\section{Biofilm Formation}

Biofilms were grown on the surface of sterile polystyrene microplates (96- or 24-well), on central venous catheter (CVC) sections or on glass-bottom petri dishes, as previously described (Vila et al., 2015). Briefly, a standardized suspension $\left(10^{7}\right.$ conidia/ml) was added to each well of the microplate, and the plates were then incubated at $36^{\circ} \mathrm{C}$ for $1.5 \mathrm{~h}$ (adherence phase). After adherence, the supernatant containing the non-adherent cells was removed, and fresh RPMI 1600 media (Sigma-Aldrich, United States) supplemented with 2\% glucose and 20\% fetal bovine serum (FBS, Gibco, United States) was added to each well. The microplates were then incubated for $30 \mathrm{~min}$ or $2,4,6,8,12,24,48$, or $72 \mathrm{~h}$. At each time point during incubation, biofilm formation was quantified using two different approaches.

\section{Biofilm Quantification Assays}

Biofilm formation on the surface of polystyrene microplates was quantified using the following two different methods: the overall biomass, which comprised both cells and the ECM, was quantified using crystal violet assays, while the metabolic activity of the cells inside the biofilms, which reflects viability and cell density, was quantified using XTT-reduction assays (Mello et al., 2016).

Crystal violet assay: At each incubation time point, the biofilms were washed with sterile PBS ( $\mathrm{pH}$ 7.2) to remove any un-adherent cells. The remaining biofilm was fixed in methanol for $15 \mathrm{~min}$ and then stained with crystal violet $(0.02 \%)$ for $20 \mathrm{~min}$. The solution was discarded, and the biofilms were washed two times with PBS. The impregnated crystal violet was dissolved using a 33\% acetic acid solution for $5 \mathrm{~min}$, and the colored solution was transferred to a clean microplate and measured using a spectrophotometer at 590 nm (SpectraMAX 340 Tunable; Molecular Devices Ltd., United States).

XTT assay: The biofilms were quantified using a XTTreduction assay, as previously described (Pierce et al., 2008). First, 
TABLE 1 | The strains used in this study.

\begin{tabular}{|c|c|c|c|}
\hline Strains obtained from the CBS collection & Origin & Isolation location & Country \\
\hline Scedosporium (Pseudallescheria) boydii CBS 117410 & Environmental & Garden soil & Spain \\
\hline Scedosporium (Pseudallescheria) boydii CBS 117432 & Clinical & Sputum of a patient with cystic fibrosis & France \\
\hline Scedosporium (Pseudallescheria) boydii CBS 120157 & Clinical & The lung of a patient with leukemia & France \\
\hline Pseudallescheria ellipsoidea CBS 301.79 & Environmental & Dung of a cow & Netherland \\
\hline Pseudallescheria angusta CBS 254.72 & Environmental & Sewage half of a digestion tank & United States \\
\hline Scedosporium aurantiacum CBS 116910 & Clinical & Ankle ulcer & Spain \\
\hline Scedosporium aurantiacum CBS 136046 & Clinical & The lung of a patient with an invasive infection & Australia \\
\hline Scedosporium aurantiacum CBS 136047 & Environmental & Environmental soil & Australia \\
\hline Scedosporium aurantiacum CBS 136049 & Environmental & Soil from a park and playground & Austria \\
\hline
\end{tabular}

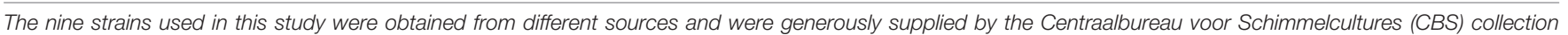
culture.

the biofilms were washed with sterile PBS, and then $100 \mu \mathrm{l}$ of a XTT: Menadione solution $(0.5 \mathrm{mg} / \mathrm{ml}: 1 \mu \mathrm{M})$ was added to the cells. The microplate was incubated for $2 \mathrm{~h}$ at $36^{\circ} \mathrm{C}$ while protected from light. Finally, the colored solution was transferred to a clean microplate, and its absorbance was measured at $490 \mathrm{~nm}$ using a spectrophotometer (SpectraMAX 340 Tunable; Molecular Devices Ltd., United States).

\section{Germination Assay}

A standardized suspension containing $10^{5}$ conidia was added to each well of a microplate with RPMI 1640, and the plates were then incubated at $36^{\circ} \mathrm{C}$ for $2,4,6$, and $12 \mathrm{~h}$. At each time point, a total of 200 cells were counted using an optical microscope, and the percentage of germinated cells was calculated.

\section{Scanning Electron Microscopy (SEM)}

The biofilms were grown on the surface of sterile CVC sections $(0.5 \mathrm{~cm})$ that were cut longitudinally cut to expose the interior. A catheter section was placed in each well of a 96-well microplate, and biofilms were formed on the inner CVC surface for 24 or $48 \mathrm{~h}$ as described above. Then, catheter sections that contained biofilms were processed for scanning electron microscopy (SEM) as previously described (Vila et al., 2013). Briefly, the CVCs were washed in $0.01 \mathrm{M}$ PBS ( $\mathrm{pH} 7.2$ ) and fixed in $2.5 \%$ glutaraldehyde and $4 \%$ formaldehyde in $0.1 \mathrm{M}$ cacodylate buffer for $1 \mathrm{~h}$ at room temperature. Subsequently, the CVCs were washed in the same buffer, post-fixed in $1 \%$ osmium tetroxide and $1.25 \%$ potassium ferrocyanide for $30 \mathrm{~min}$, and dehydrated in a series of ethanol solutions with increasing concentrations $(30,50,70,90,100 \%$ and 'ultra-dry' ethanol) for $30 \mathrm{~min}$ at each concentration. Then, the samples were critical point-dried in $\mathrm{CO}_{2}$, coated with gold and observed using a FEI Quanta 250 scanning electron microscope (FEI, Netherlands).

\section{Confocal Laser Scanning Microscopy (CLSI)}

Biofilms were formed on glass-bottom petri dishes (CellView ${ }^{\mathrm{TM}}$, Greiner Bio-One, Germany) as described above. Then, after the biofilms were gently fixed in a $2 \%$ formaldehyde solution, they were incubated with the following fluorescent markers: Concanavalin A conjugated to Alexa Fluor 488,
Filmtracer ${ }^{\circledR}$ Sypro Ruby (both from Molecular Probes, Invitrogen, United States), and Calcofluor White M2R (Sigma-Aldrich, United States). The biofilms were observed using a Leica TCS-SPE confocal scanning microscope (Leica, Germany), and Z-stack reconstructions were analyzed using Fiji software (Schindelin et al., 2012).

\section{Antifungal Susceptibility Assay}

Susceptibility to antifungal drugs was evaluated according to the EUCAST protocols, with some modifications (Taj-Aldeen et al., 2016). Briefly, to each well of a microplate, $100 \mu l$ of a standardized suspension $\left(2 \times 10^{5}\right.$ conidia/ml $)$ was added to $100 \mu \mathrm{l}$ of antifungal drugs serially diluted in RPMI 1640 (supplemented with $2 \%$ glucose and buffered with 3-(Nmorpholino) propanesulfonic acid (MOPS) $0.165 \mathrm{~mol} / \mathrm{l}, \mathrm{pH} 7.0$ ). The concentrations of caspofungin, fluconazole, itraconazole and voriconazole ranged from 0.125 to $128 \mu \mathrm{g} / \mathrm{ml}$. Control cells were grown in the presence of DMSO (for itraconazole and voriconazole) or water (for caspofungin and fluconazole). The plates were incubated at $36^{\circ} \mathrm{C}$ for $72 \mathrm{~h}$.

To evaluate biofilm susceptibility, biofilms were formed as previously described (Pierce et al., 2008). Antifungal drugs were added to $24 \mathrm{~h}$-old biofilms at the same concentration range described for planktonic cells. The plates were then incubated for an additional $24 \mathrm{~h}$ at $36^{\circ} \mathrm{C}$. For both susceptibility tests (planktonic cells and biofilms), a visual reading was performed to define the minimal inhibitory concentration (MIC), and the results were confirmed using a XTT-reduction assay as described above.

\section{In Vivo Assay with Galleria mellonella}

A survival analysis was performed using the larvae of G. mellonella as described by de Lacorte Singulani et al. (2016). Each group of larvae (10 larvae per group) was infected with $10 \mu \mathrm{l}$ of $10^{6}$ conidia via the last left pro-leg using a Hamilton syringe. The S. boydii CBS 120157, S. boydii CBS 117410, S. aurantiacum CBS 136046 and S. aurantiacum CBS 136047 strains were selected for these experiments. A group of uninfected larvae and a group of uninfected larvae that were inoculated with PBS were used as controls. The larvae were incubated in Petri dishes at 




Scedosporium boydi CBS 117410

CBS 117432

A. Scedosporium boydii CBS 120157

\section{C}
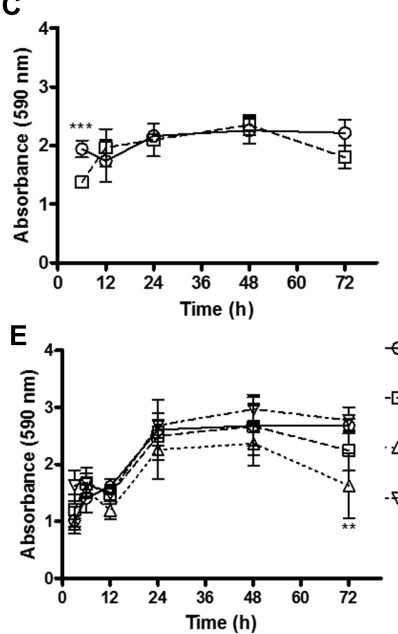

๑ Pseudallescheria ellipsoidea

-๒. Pseudallescheria

Scedosporium aurantiacum CBS 116910

Scedosporium aurantiacum CBS 136046

Scedosporium aurantiacum CBS 136047

Scedosporium aurantiacum CBS 136049
Scedosporium boydii

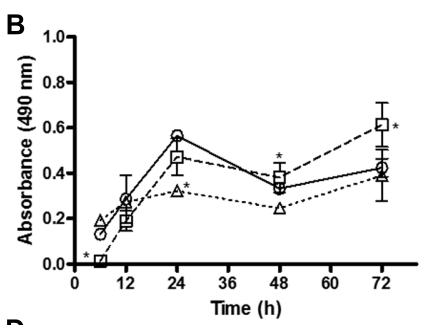

D

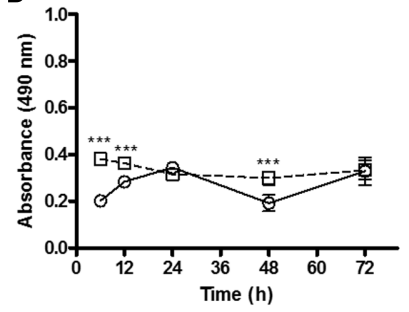

$\mathbf{F}$

FIGURE 1 | The kinetics of biofilm formation was evaluated using biomass and metabolic assays. Biofilm formation was monitored and quantified using two complementary methods at selected time points $(3,6,12,24,48$, and 72 h). The graphs (A,C,E) shown demonstrate the total biomass of the biofilms. The results were quantified using a crystal violet assay. The graphs shown in (B,D,F) demonstrate the metabolic activity of the cells inside the biofilms. The results were quantified using XTT-reduction assays. Statistical differences $(P<0.05)$ are represented by asterisks. *Statistical difference when S. boydii CBS 117410 was used as a reference. ${ }^{*}$ Statistical difference when $S$. aurantiacum CBS 136049 was used as a reference. ${ }^{* * *}$ Statistical difference when $P$. angusta as was used as a reference.

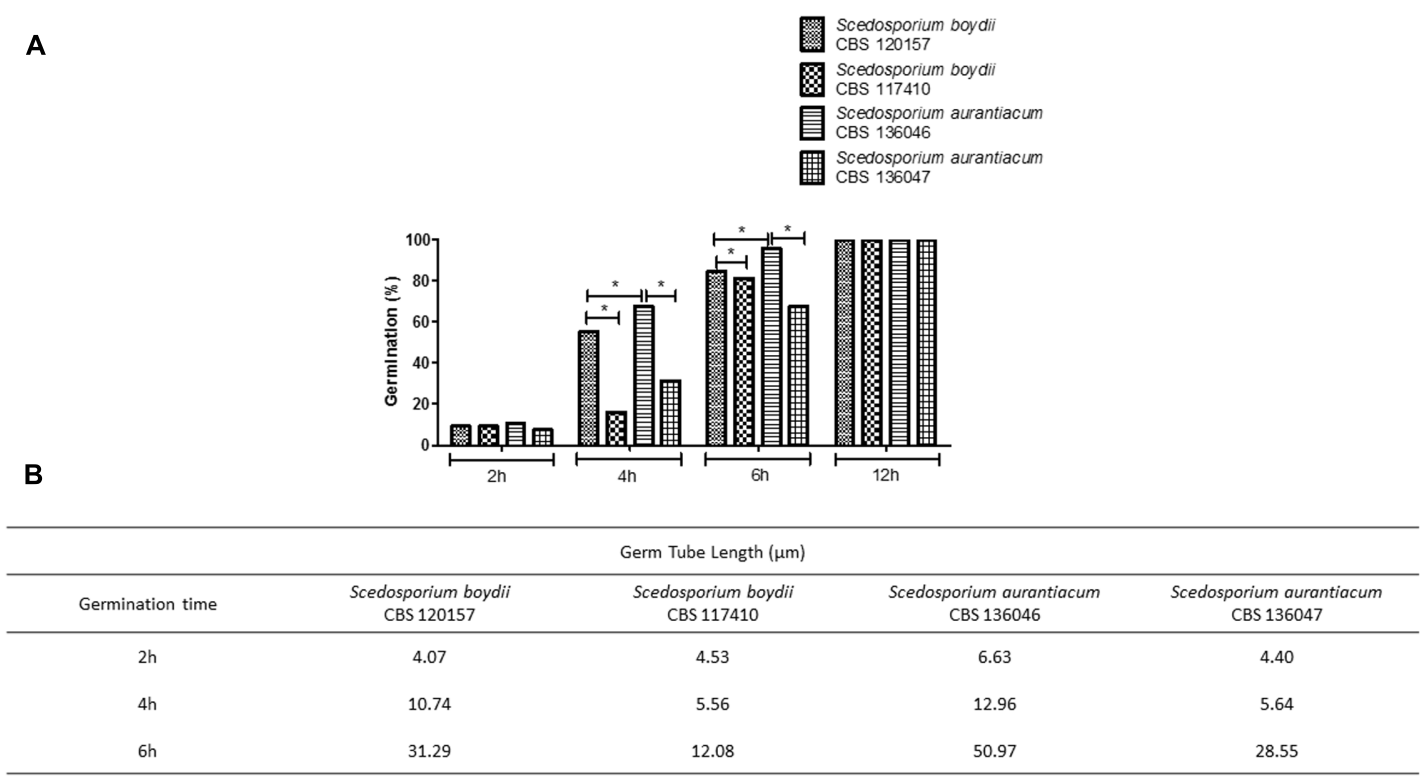

FIGURE 2 | Germ tube formation in clinical and environmental strains of Scedosporium boydii and S. aurantiacum. (A) The germination process was monitored over time and quantified by counting the percentage of germinated cells at selected time points (2, 4, 6, and 12 h). (B) Germ tube length was monitored during the germination process at selected time points $(2,4$, or 6 h). Statistical differences $(P<0.05)$ are represented by asterisks. 


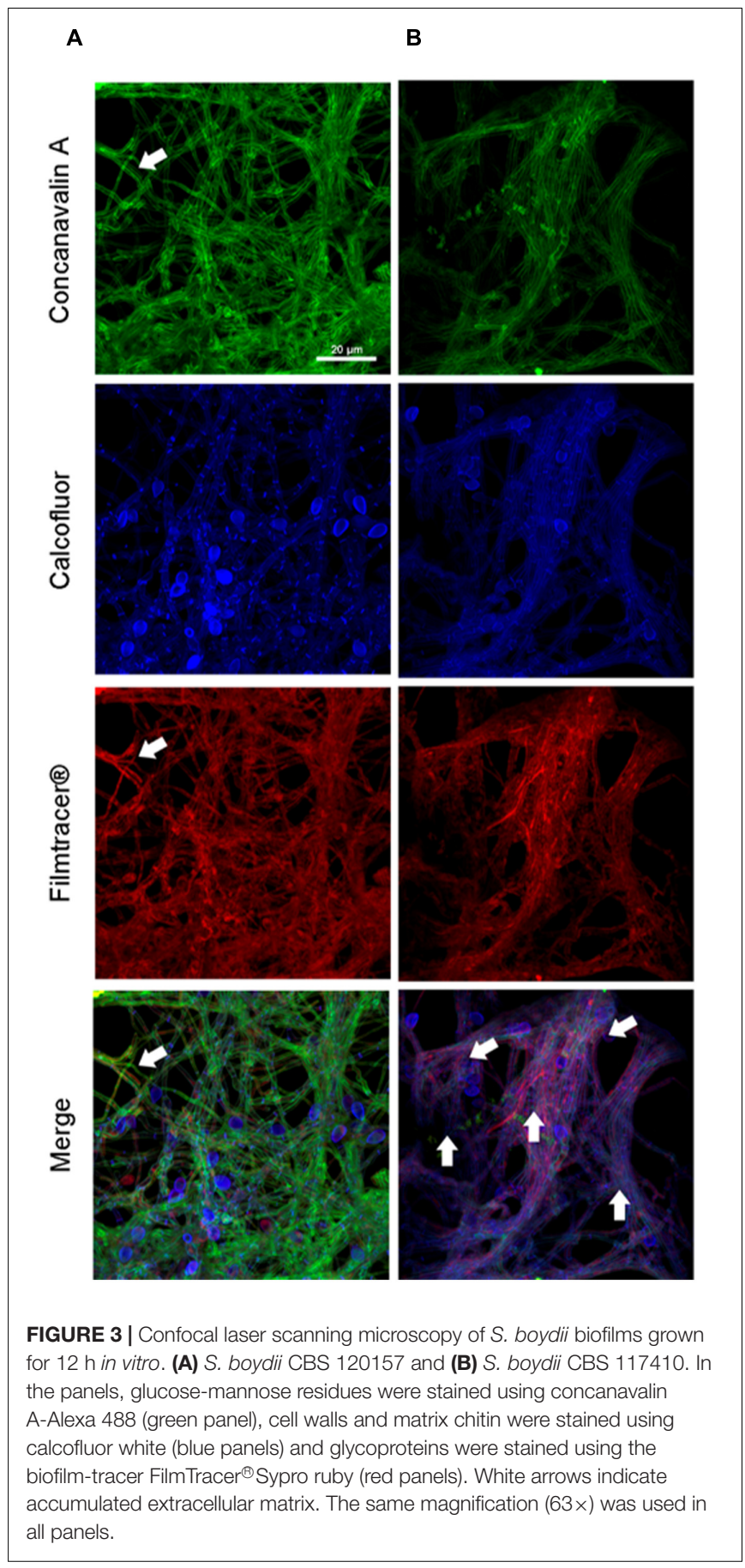

$37^{\circ} \mathrm{C}$, and population death was evaluated daily for a total of 9 days.

\section{Statistical Analysis}

All statistical analysis were performed using GraphPad Prism 6.0 software (GraphPad, United States). A variance two-way ANOVA was performed using Tukey's and Bonferroni's comparisons tests to evaluate the kinetics of biofilm formation in addition to the germination process and survival experiments.

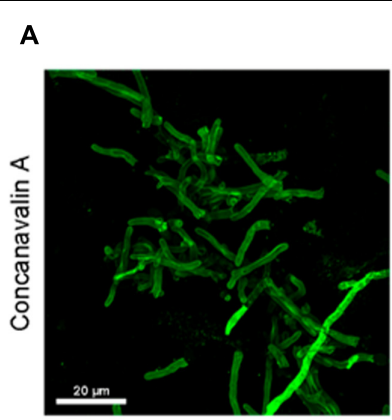

B
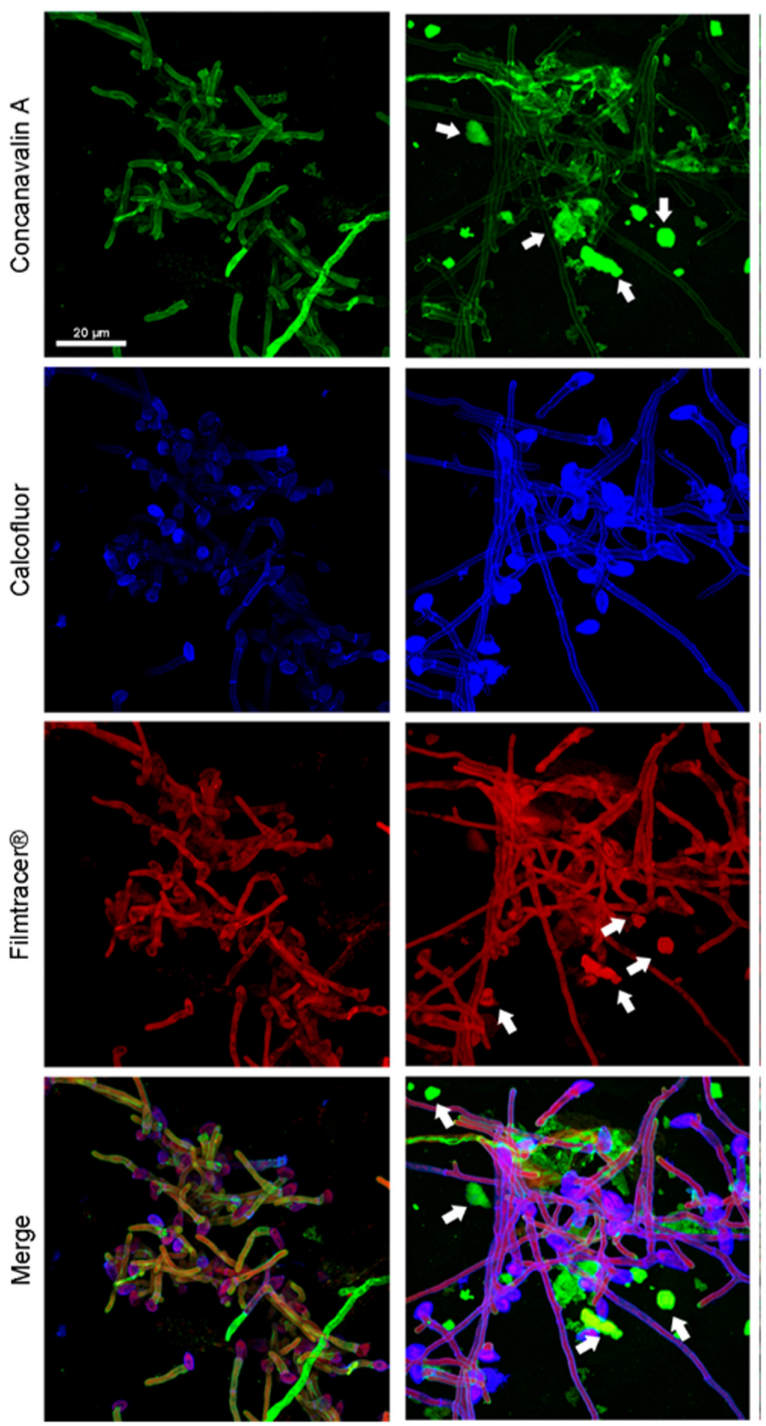

FIGURE 4 | Confocal microscopy of a $S$. aurantiacum biofilms grown for $6 \mathrm{~h}$ in vitro. (A) S. aurantiacum CBS 136046 and (B) S. aurantiacum CBS 136047. In the panels, glucose-mannose residues were stained using concanavalin A-Alexa 488 (green panel), cell walls and matrix chitin were stained using calcofluor white (blue panels) and glycoproteins were stained using the biofilm-tracer FilmTracer ${ }^{\circledR}$ Sypro ruby (red panels). White arrows indicate accumulated extracellular matrix. The same magnification $(63 \times)$ was used in all panels.

\section{RESULTS}

\section{Kinetics of Biofilm Formation}

The growth kinetics of all nine strains that formed biofilms on polystyrene microplates were analyzed by correlating the results of two different analyses: the total biofilm biomass (including cells, both dead and alive, and extracellular matrix), which was evaluated using crystal violet assays (Figures 1A,C,E) and metabolic activity, which was quantified using XTT-assays 

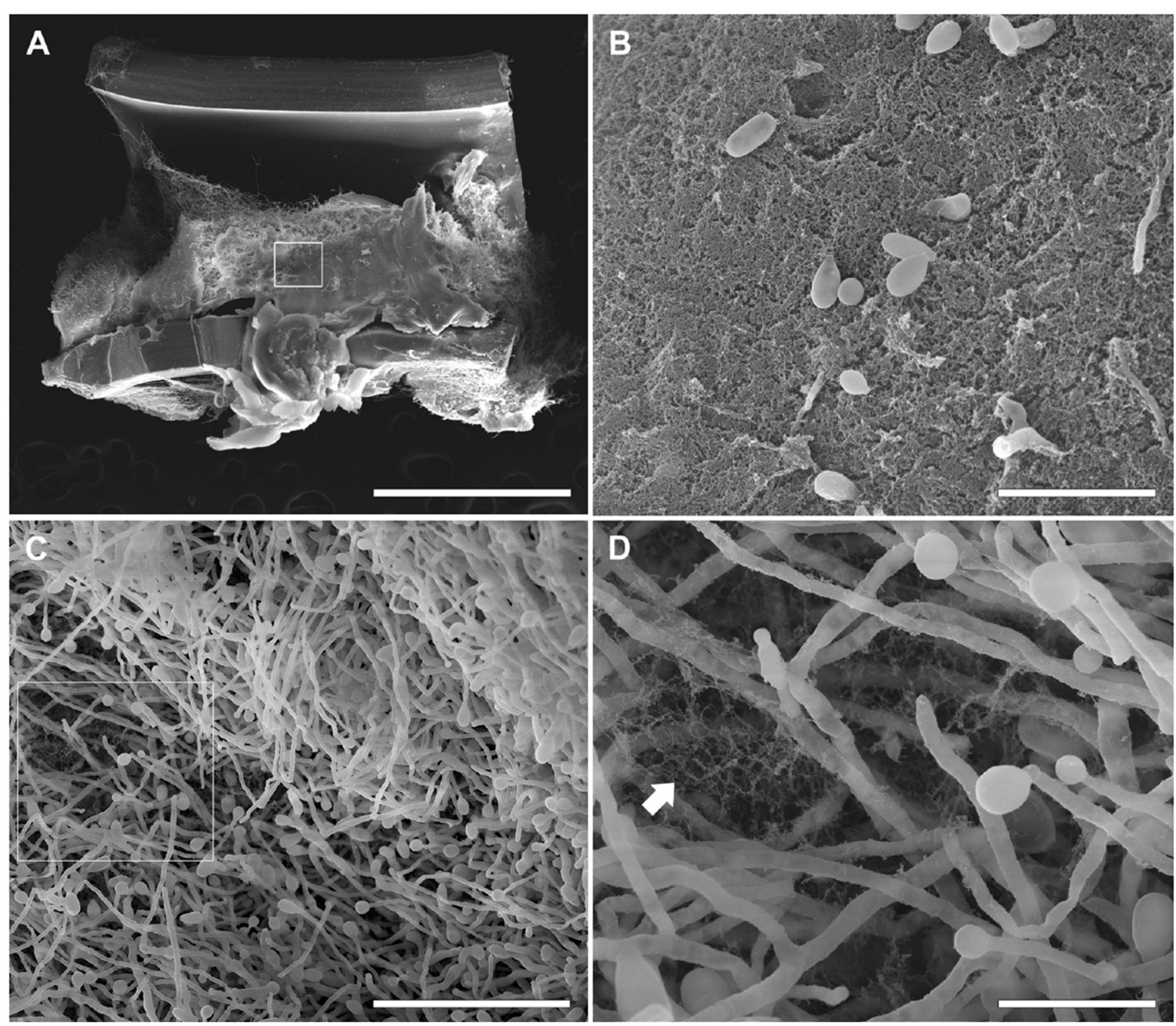

FIGURE 5 | Scanning electron microscopy of S. boydii clinical isolate (CBS 120157) biofilms grown on central venous catheters (CVCs). Biofilms were grown in vitro on the inner surface of $0.5 \mathrm{~cm}$ sections of sterile CVCs for $48 \mathrm{~h}$. (A) A dense mass of $S$. boydii biofilm covered most of the CVC surface. (B) The inset of the white square shown in panel (A) shows an area of dense extracellular matrix (ECM; white arrow) and a dispersion of conidia. (C) A representative area of the internal biofilm. (D) An inset of the white square in panel. (C) showing a network of hyphae with ECM residues. Bars correspond to $1 \mathrm{~mm}$ (A), $20 \mu \mathrm{M}$ (B), $50 \mu \mathrm{M}$ (C), and $10 \mu \mathrm{M}$ (D)

(Figures $\mathbf{1 B}, \mathbf{D}, \mathbf{F})$. These nine strains are representative isolates defined on a meeting of the Working Group on Pseudallescheria/Scedosporium Infections, whose researchers received the samples to generate more data about these fungi.

In our analysis of biofilm biomass, among the three $S$. boydii strains, the clinical isolate obtained from human lungs ( $S$. boydii CBS 120157) grew significantly faster than the other two isolates (CBS 117432 and CBS 117410), especially at 6 and $12 \mathrm{~h}$ (Figure 1A). The environmental strain (S. boydii CBS 117410) was the slowest-growing strain, and it reached the same biomass as S. boydii CBS 120157 only after $48 \mathrm{~h}$. These results suggest that the clinical $S$. boydii strains form biofilms faster than the environmental strain but that all three strains were able to form thick biofilms after $48 \mathrm{~h}$ (Figure 1A). Interestingly, the S. boydii clinical strain (CBS 120157) had significantly lower metabolic activity inside the biofilm at $24 \mathrm{~h}$, but at $48 \mathrm{~h}$, all three $S$. boydii strains had similar metabolic activity (Figure 1B). The typical environmental species $P$. ellipsoidea and $P$. angusta produced a similar amount of biomass and $P$. angusta exhibited a significantly higher metabolic rate at 6, 12, and $48 \mathrm{~h}$ (Figures 1C,D).

The $S$. aurantiacum clinical isolates shared a similar pattern of biomass growth, and both strains produced biomass levels that were the same as that produced by the $S$. boydii clinical isolates within $24 \mathrm{~h}$ (Figure 1E). Remarkably, across all of the $S$. aurantiacum strains, there was no significant difference in the amount of biomass produced or metabolic activity rates, excepting at $72 \mathrm{~h}$ when S. aurantiacum CBS 136047 presented significantly less biomass comparing to the other strains (Figures 1E,F).

\section{Conidial Germination Assay}

For the further analysis, two strains of S. aurantiacum and $S$. boydii were chosen, since the experiments present a significantly high cost to be performed with all nine isolates. A germination assay was performed using fresh conidia. One clinical and one environmental strain of each species (S. boydii CBS 120157 and CBS 117410 and S. aurantiacum CBS 136046 and 

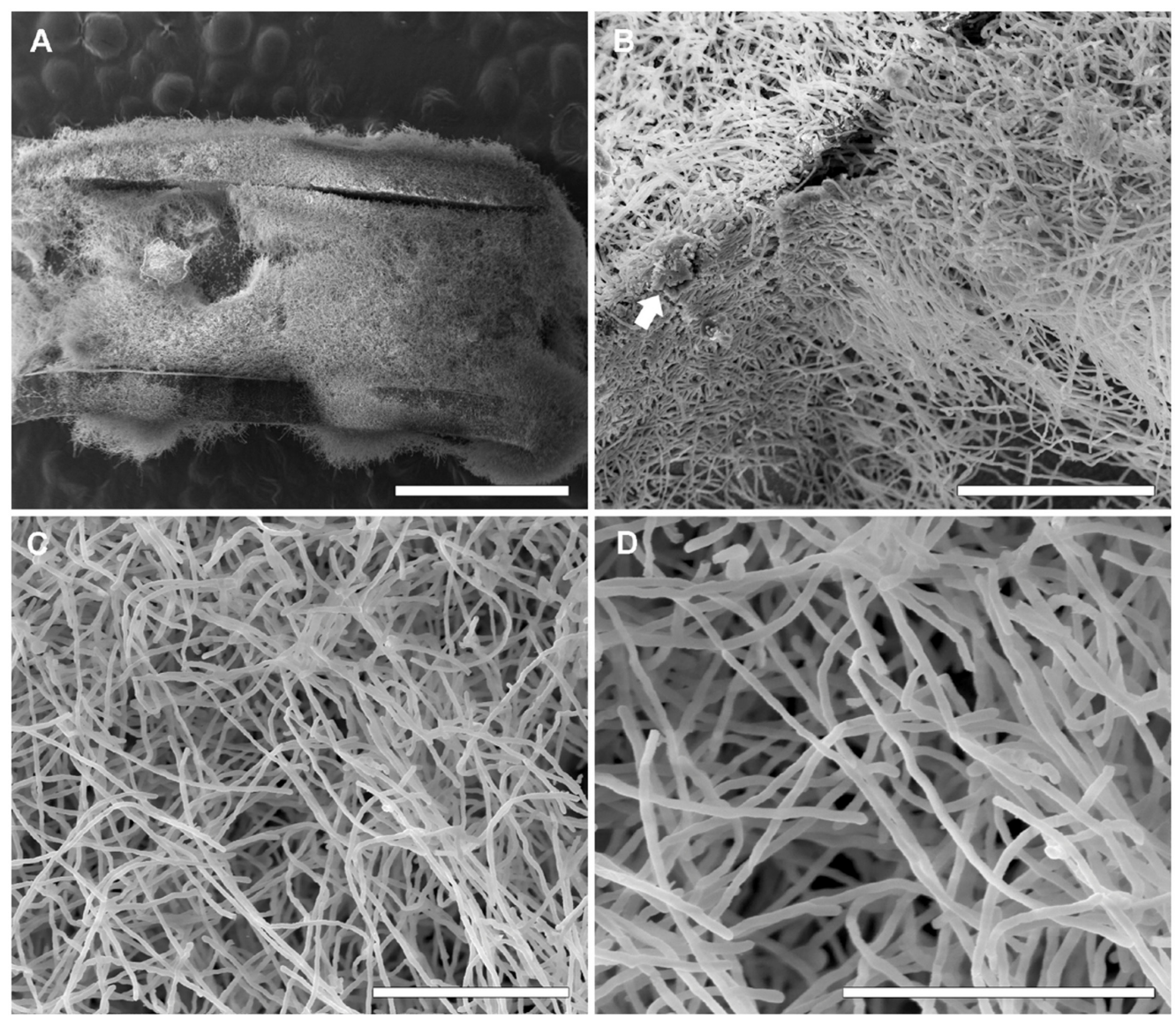

FIGURE 6 | Scanning electron microscopy of S. aurantiacum clinical isolate (CBS 136046) biofilms grown on CVCs. Biofilms were grown in vitro on the inner surface of $0.5 \mathrm{~cm}$ sections of sterile CVCs for $24 \mathrm{~h}$. (A) A dense mass of S. aurantiacum biofilms covered the entire CVC surface. (B,C) A representative area of an internal area of a biofilm with a dense network of hyphae (white arrow). (D) A higher magnification of the central area shown in panel (C). The bars correspond to $1 \mathrm{~mm}$ (A), $100 \mu \mathrm{M}$ (B), $50 \mu \mathrm{M}$ (C), and $40 \mu \mathrm{M}$ (D)

CBS 136047) were selected to evaluate growth profiles (Figure 2). For all of the included strains, regardless of the germ tube length, approximately $10 \%$ of the cells were germinating after $2 \mathrm{~h}$ of incubation, and 100\% were germinating after $12 \mathrm{~h}$ (Figure 2A). The most important difference between the germination profiles was observed after 4 and $6 \mathrm{~h}$ of incubation when the clinical isolates, S. boydii CBS 120157 and S. aurantiacum CBS 136046, were found to have germinated 2- to 3-fold faster than the environmental isolates, S. boydii CBS 117410 and S. aurantiacum CBS 136047 (Figure 2A). Similar results were observed when the germ tube lengths were measured, as follows: although similar germ tube lengths were observed after $2 \mathrm{~h}$ of incubation, the tube lengths were different after $4 \mathrm{~h}$ of incubation (Figure 2B).

\section{Confocal Scanning Microscopy of Scedosporium/Pseudallesheria Biofilms}

To compare the biofilm structures of clinical and environmental strains of $S$. boydii and S. aurantiacum, we selected one clinical and one environmental strain of each species (S. boydii 120157 and 117410, respectively, and S. aurantiacum 136046 and 136047, respectively) and imaged the biofilms using CLSM. Biofilm cells were stained with (a) Concanavalin A, which stains glucose and mannose residues, both of which are present in cell walls and biofilm ECM; (b) Calcofluor white, which stains chitin structures in the cell walls of fungi, and (c) Filmtracer ${ }^{\circledR}$ sypro ruby, which stains glycoproteins known to be abundant in the ECM of other fungi.

A more robust biofilm was formed by the $S$. boydii clinical isolate (120157) than the corresponding environmental strain (117410) (Figures 3A,B). These results corroborate the data shown in Figures 1, 2. However, there was little to no visible difference between the biofilms formed by the clinical and the environmental strains of S. aurantiacum (Figures 4A,B).

\section{Scanning Electron Microscopy of Scedosporium/Pseudallesheria Biofilms}

Biofilms were formed on catheters using the clinical isolates of each species (i.e., S. boydii CBS 120157 and S. aurantiacum CBS 136046) with the aim of evaluating their ability to adhere to and develop biofilms on medical devices. The biofilms were visualized using SEM. 
Similar to the results previously described for the biofilms grown on polystyrene and glass-bottom surfaces in this study, biofilms formed faster on CVCs in the S. aurantiacum clinical isolate than in the $S$. boydii clinical isolate. Whereas $24 \mathrm{~h}$ was sufficient for $S$. aurantiacum to colonize the entire catheter surface (Figure 6), the $S$. boydii isolate required $48 \mathrm{~h}$ to reach a similar biomass density (Figure 5). The presence of ECM was observed among hyphae that had adhered to the catheter surface, especially in $S$. boydii and less in S. aurantiacum, suggesting the formation of a mature biofilm structure (arrows in Figures 5D, 6B).

\section{Biofilm Formation on Different Abiotic Surfaces}

To confirm that $S$. boydii and $S$. aurantiacum grow in a similar manner on all of the different surfaces used in this study (i.e., polystyrene, glass bottom dishes and catheters), biofilm formation was simultaneously measured using crystal violet and XTT-reduction assays in clinical isolates of S. boydii (CBS 120157) and S. aurantiacum (CBS 136046) that were grown on all three surfaces. After $24 \mathrm{~h}$, both isolates had adhered to and grown biofilms on all three surfaces and, as expected, faster growth was observed on all 3 surfaces by $S$. aurantiacum as compared with S. boydii (Figure 7).

\section{Antifungal Susceptibility of Biofilms and Planktonic Cells}

Because increased resistance to drugs is the most clinically relevant phenotypical alteration observed in biofilms, we evaluated the susceptibility of $S$. boydii and $S$. aurantiacum strains to different antifungal drugs and compared the results to those obtained for their planktonic counterparts (primary suspension inoculums were used to form the biofilms). As expected, the biofilms were generally less susceptible to all the azole drugs $(\mathrm{MIC}>128 \mu \mathrm{g} / \mathrm{ml}$ ) than were their planktonic cells. Interestingly, caspofungin was the only drug for which we obtained a lower MIC value in the biofilms $(32 \mu \mathrm{g} / \mathrm{ml})$ than in the planktonic cells $(64 \mu \mathrm{g} / \mathrm{ml})$ (Table 2$)$.

In the planktonic cells, itraconazole (MIC, 1-2 $\mu \mathrm{g} / \mathrm{ml}$ ) and voriconazole (MIC, $0.5-1 \mu \mathrm{g} / \mathrm{ml}$ ) were the most active antifungals. As was expected for filamentous fungi, these isolates were less sensitive to fluconazole (MIC between 16 and $64 \mu \mathrm{g} / \mathrm{ml}$ ) (Table 2). No relevant difference was observed in antifungal susceptibility between these distinct genera and or by sample source.

\section{In Vivo Virulence Assay Using Galleria mellonella}

An in vivo infection model was established using larvae of G. mellonella. In this assay, we evaluated the pathogenicity of the strains used in this study to construct survival curves (Figure 8). Interestingly, the $S$. aurantiacum clinical isolate (136046), which had the fastest germ tube elongation (Figure 2B), was also the most virulent strain in that it had caused $100 \%$ mortality in G. mellonella at 6 days after infection (Figure 8). The other three strains (S. aurantiacum 136047, P. boydii 120157 and S. boydii 117410) had similar virulence patterns and had killed the larvae by 8-9 days post-infection (Figure 8).

\section{DISCUSSION}

The ability of some Pseudallescheria/Scedosporium species, such as $S$. apiospermum, S. aurantiacum, S. minutisporum, and L. prolificans, to form biofilms was recently described by Mello et al. (2016). In this study, Mello et al. reported that both species can adhere to polystyrene surfaces and lung epithelial cells, on which they form biofilms that are less susceptible to antifungal drugs. Additionally, the authors

TABLE 2 | The minimum inhibitory concentration (MIC) of different antifungal drugs used to treat S. boydii and S. aurantiacum planktonic cells and biofilms.

\begin{tabular}{|c|c|c|c|}
\hline Fungi & Antifungal drugs (range 128-0.125 $\mu \mathrm{g} / \mathrm{ml}$ ) & MIC of planktonic cells $(\mu \mathrm{g} / \mathrm{ml})$ & MIC of biofilms $(\mu \mathrm{g} / \mathrm{ml})$ \\
\hline Scedosporium (Pseudallescheria) & Caspofungin & 64 & 32 \\
\hline \multirow[t]{3}{*}{ boydii CBS 120157} & Fluconazole & 32 & $>128$ \\
\hline & Itraconazole & 2 & $>128$ \\
\hline & Voriconazole & 1 & $>128$ \\
\hline Scedosporium (Pseudallescheria) & Caspofungin & 64 & 32 \\
\hline \multirow[t]{3}{*}{ boydii CBS 117410} & Fluconazole & 64 & $>128$ \\
\hline & Itraconazole & 2 & $>128$ \\
\hline & Voriconazole & 0.5 & $>128$ \\
\hline Scedosporium aurantiacum CBS & Caspofungin & 64 & 32 \\
\hline \multirow[t]{3}{*}{136046} & Fluconazole & 16 & $>128$ \\
\hline & Itraconazole & 1 & $>128$ \\
\hline & Voriconazole & 0.5 & $>128$ \\
\hline Scedosporium aurantiacum CBS & Caspofungin & 64 & 32 \\
\hline \multirow[t]{3}{*}{136047} & Fluconazole & 32 & $>128$ \\
\hline & Itraconazole & 1 & $>128$ \\
\hline & Voriconazole & 0.5 & $>128$ \\
\hline
\end{tabular}

An antifungal susceptibility test was performed using planktonic cells and pre-formed biofilms. 

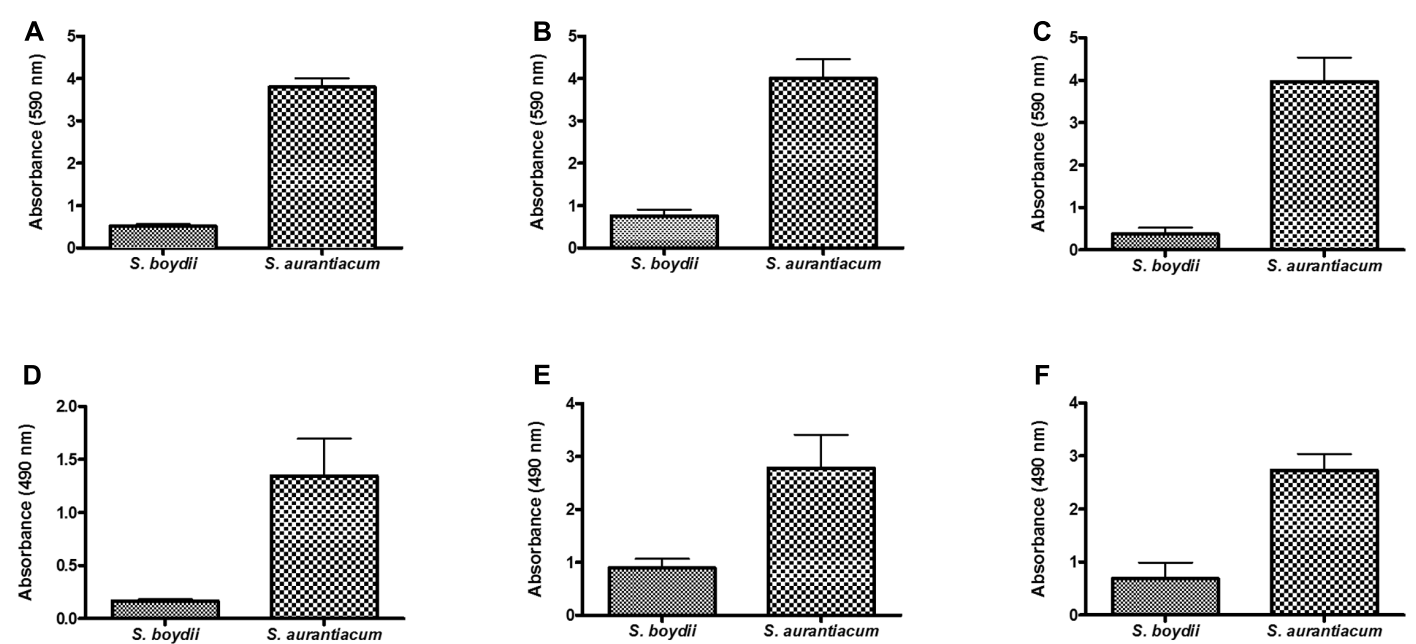

FIGURE 7 | Comparison of biofilm formation by S. boydii and S. aurantiacum on three different surfaces. Biofilm formation was evaluated after 24 h of growth. The total biomass of the biofilms was quantified using a crystal violet assay (A,B,C), and the metabolic activity of the cells inside the biofilms was quantified using a XTT-reduction assay (D,E,F). Three different surfaces were used, including glass Petridish bottoms (A,D), polystyrene (B,E), and catheters (C,F). Statistical differences $(P<0.05)$ are represented by asterisks.

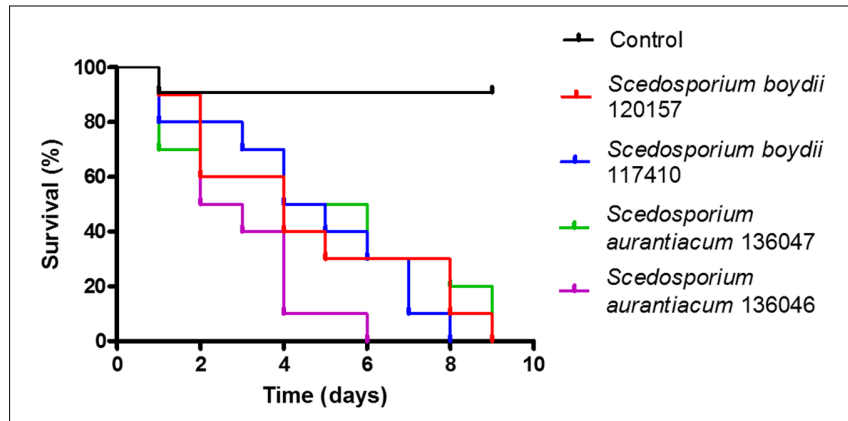

FIGURE 8 | In vivo infection model of Galleria mellonella. An in vivo infection model was performed in which we infected 10 larvae per group with $10^{6}$ conidia of one of the strains used in this study. The control group was inoculated with PBS. Each population was observed daily for 9 days. The survival results are shown. Statistical differences $(P<0.05)$ are represented by asterisks. characterized biofilm organization, and their results indicated that the clinically relevant Pseudallescheria/Scedosporium species formed biofilms. In the present study, we compared the ability of clinical and environmental isolates of two Scedosporium species to germinate and grow as biofilms in vitro on different surfaces. The correlation between biofilm formation and antifungal susceptibility or virulence was also examined.

Our results show that biofilm formation is faster in S. aurantiacum than in $S$. boydii (Figures 1, 7), as previously described by Mello et al. (2016). Interestingly, the metabolic activity of the $S$. boydii biofilms was higher than that of the S. aurantiacum strains (Figure 1). Because the metabolic activity of the biofilms formed by all of the tested strains was not altered during the course of the kinetics assay, and because our visual observations showed that there was an increase in fungal growth, we hypothesized that the abundant secretion of ECM in biofilms could impair XTT penetration, resulting in a reduced color change reaction that may have compromised the accuracy of the assay.

After $12 \mathrm{~h}$, the S. boydii clinical isolate (CBS 120157) had formed thicker biofilms than was observed in the environmental strain (CBS 117410), as demonstrated by the kinetics assay and CLSM (Figures 1, 3). Interestingly, according to CLSM, after $6 \mathrm{~h}$ the clinical isolate of $S$. aurantiacum (CBS 136046) had formed less biofilm than was observed in the environmental isolate (CBS 136047) (Figure 4). However, the presence of ECM among the interconnected hyphae was more evident in the CLSM images of the clinical isolate (CBS 136046) (Figure 4). In S. boydii (CBS 120157), ECM could be well observed in SEM pictures (Figure 5). In the biofilms of both species, ECM glycoproteins were homogeneously distributed around the mycelial mass and some glucose-rich residues. The ECM is a crucial structure for robust biofilms that contributes to the increase in resistance observed in these communities (Borghi et al., 2016). Therefore, determining the composition of the ECM is very important for understanding and predicting drug-resistance in biofilms. The ECM composition varies among different fungal species and even across different environmental conditions under which biofilm can grow (Al-Fattani and Douglas, 2006; Flemming and Wingender, 2010; Reichhardt et al., 2015). As far as we are aware, the ECM composition of Pseudallescheria and Scedosporium biofilms is unknown, and further studies are therefore needed to determine its composition and whether it plays an important role in drug-sequestration, as was described in Candida biofilms (Nett et al., 2010; Vediyappan et al., 2010; Mitchell et al., 2016).

Biofilm formation is an important risk factor for mortality in patients with bloodstream infections of $C$. albicans 
(Rajendran et al., 2016), especially because these biofilms can grow on the surface of any inserted devices and thereby create a continuous cycle of reinfection. Reports showing that $S$. boydii and $S$. apiospermum caused catheter-related infections have been published (Perez et al., 1988; Eldin et al., 2012). Here, we used SEM to demonstrate that $S$. boydii and $S$. aurantiacum clinical isolates obtained from lung infections can form robust biofilms in vitro on CVC surfaces (Figures 5, 6). SEM images showed that a multicellular biofilm had formed and that it was composed of a dense network of hyphae on which several conidia were ready to be released from the end of mycelia. In addition, the presence of ECM among the mycelia was also observed, and these data corroborated those found in the CLSM analysis. The biofilms formed by $S$. boydii and S. aurantiacum inside the CVCs were very similar to those previously reported for Aspergillus sp. (Kaur and Singh, 2014) and other Pseudallescheria/Scedosporium species (Mello et al., 2016). These data show that Pseudallescheria/Scedosporium species are capable of forming biofilms on non-biotic surfaces, which reinforces their potential to cause opportunistic infections in healthcare units.

Fungal germination is the initial step in mycelia and biofilm formation and, therefore, the germination rate of each species might be important for predicting its biofilm formation profile. As expected, both clinical isolates germinated faster than their environmental counterparts, and the $S$. aurantiacum clinical isolate was the fastest strain to germinate. To our knowledge, this is the first study to compare biofilm formation and germination using different clinical and environmental isolates obtained from the Pseudallescheria/Scedosporium species. Similar results were previously demonstrated by Nunes et al. (2013) in Rhodotorula mucilaginosa, an emerging yeast pathogen that causes invasive infections and in which the clinical isolates were more capable of forming biofilms than were their environmental isolates (Nunes et al., 2013). In Candida spp., the formation of biofilms by clinical isolates was associated with higher mortality in patients with candidemia and was considered a significant predictor of mortality in hospitalized patients (Tumbarello et al., 2007; Rajendran et al., 2016). Conversely, an S. aurantiacum clinical isolate was found to be more virulent in vivo than an environmental strain of this species (Figure 8). In fact, this isolate had the highest virulence of all strains tested and had killed $90 \%$ of the population in 4 days. The clinical isolate of $S$. aurantiacum also produced a more substantial biofilm than was produced by the clinical isolate of $S$. boydii and was the fastest strain to germinate, suggesting that there is a correlation between the speed of germination, biofilm formation and virulence.

Biofilms are structures that have well-known associations with increased levels of antifungal resistance (Borghi et al., 2015). Here, we show that Scedosporium biofilms are less susceptible to all azoles than are their planktonic counterparts (Table 2). The role of biofilms in antifungal resistance has been extensively studied in Aspergillus and Candida.
It is widely accepted that the glucan-enriched ECM of C. albicans biofilms sequesters antifungal drugs and reduces susceptibility to them (Nett et al., 2010). Additionally, efflux pumps have been identified in both $C$. albicans and Aspergillus biofilms, in which they lead to resistance to azoles (Rajendran et al., 2011; Ramage et al., 2011). Hence, further studies are needed to identify the mechanisms involved in the increased resistance of Pseudallescheria/Scedosporium biofilms.

In summary, our results demonstrate that clinical and environmental strains of Pseudallescheria/Scedosporium form biofilms on different abiotic surfaces and that this process is correlated with antifungal susceptibility and virulence in vivo. Because Pseudallescheria/Scedosporium species are pathogens that are increasing in frequency worldwide and because most such infections are resistant to the available antifungal arsenal, obtaining a better understanding of the role of biofilms in hospital-acquired cases of pseudallescheriosis and scedosporiosis is increasingly important. However, determining how biofilms contribute to their pathogenesis would allow us to better evaluate the effectiveness of treatments for these infections.

\section{AUTHOR CONTRIBUTIONS}

RR-P, JdM, TV, BF, and VA conceived, designed and performed the experiments. RR-P, JdM, TV, SF, SR, and EB-B analyzed the experiments. RR-P, JdM, TV, SR, and EB-B drafted the manuscript.

\section{FUNDING}

This work was supported by the Conselho Nacional de Desenvolvimento Científico e Tecnológico (CNPq), Fundação de Amparo à Pesquisa no Estado do Rio de Janeiro (FAPERJ), Coordenação de Aperfeiçoamento Pessoal de Nível Superior (CAPES-PROEX) and Universidade Federal do Rio de Janeiro.

\section{ACKNOWLEDGMENTS}

We thank Dr. Fernando Almeida from Centro Nacional de Biologia Estrutural e de Bioimagem (CENABIO-UFRJ) at Federal University of Rio de Janeiro for helping with the CLSM and Prof. Sybren de Hoog from the Centraalbureau voor Schimmelcultures (CBS) for providing the strains used in this study. All of the strains used in this study are part of the Working Group on Pseudallescheria/Scedosporium infections, which our laboratory participates in. This study is part of the International Society of Human and Animal Mycology (ISHAM). These nine strains were generously provided to different groups to generate a database of new information obtained from different areas related to this fungal species. 


\section{REFERENCES}

Al Refai, M., Duhamel, C., Le Rochais, J. P., and Icard, P. (2002). Lung scedosporiosis: a differential diagnosis of aspergillosis. Eur. J. Cardiothorac. Surg. 21, 938-939. doi: 10.1016/S1010-7940(02)00068-4

Al-Fattani, M. A., and Douglas, L. J. (2006). Biofilm matrix of Candida albicans and Candida tropicalis: chemical composition and role in drug resistance. J. Med. Microbiol. 55(Pt 8), 999-1008. doi: 10.1099/jmm.0.46569-0

Borghi, E., Borgo, F., and Morace, G. (2016). Fungal biofilms: update on resistance. Adv. Exp. Med. Biol. 931, 37-47. doi: 10.1007/5584_2016_7

Borghi, E., Morace, G., Borgo, F., Rajendran, R., Sherry, L., Nile, C., et al. (2015). New strategic insights into managing fungal biofilms. Front. Microbiol. 6:1077. doi: $10.3389 /$ fmicb. 2015.01077

Bouchara, J. P., Horre, R., and de Hoog, S. (2009). Pseudallescheria and Scedosporium: emerging opportunists. Med. Mycol. 47, 341-342. doi: 10.1080/ 13693780902756974

Cortez, K. J., Roilides, E., Quiroz-Telles, F., Meletiadis, J., Antachopoulos, C., Knudsen, T., et al. (2008). Infections caused by Scedosporium spp. Clin. Microbiol. Rev. 21, 157-197. doi: 10.1128/CMR.00039-07

Davies, D. (2003). Understanding biofilm resistance to antibacterial agents. Nat. Rev. Drug Discov. 2, 114-122. doi: 10.1038/nrd1008

de Lacorte Singulani, J., Scorzoni, L., de Paula, E. S. A. C., Fusco-Almeida, A. M., and Mendes-Giannini, M. J. (2016). Evaluation of the efficacy of antifungal drugs against Paracoccidioides brasiliensis and Paracoccidioides lutzii in a Galleria mellonella model. Int. J. Antimicrob. Agents 48, 292-297. doi: 10.1016/j.ijantimicag.2016.05.012

Eldin, C., Chiche, L., Thomas, G., Dicostanzo, M. P., Durand, J. M., Harle, J. R., et al. (2012). Scedosporium apiospermum catheter-related soft-tissue infection: a case report and review of the literature. Med. Mycol. 50, 627-630. doi: 10.3109/ 13693786.2011.639035

Fan, Z., Li, Z., Xu, Z., Li, H., Li, L., Ning, C., et al. (2015). cspA influences biofilm formation and drug resistance in pathogenic fungus Aspergillus fumigatus. Biomed. Res. Int. 2015:960357. doi: 10.1155/2015/960357

Filler, S. G., and Sheppard, D. C. (2006). Fungal invasion of normally nonphagocytic host cells. PLoS Pathog. 2:e129. doi: 10.1371/journal.ppat.0020129

Flemming, H. C., and Wingender, J. (2010). The biofilm matrix. Nat. Rev. Microbiol. 8, 623-633. doi: 10.1038/nrmicro2415

Guarro, J., Kantarcioglu, A. S., Horre, R., Rodriguez-Tudela, J. L., Cuenca Estrella, M., Berenguer, J., et al. (2006). Scedosporium apiospermum: changing clinical spectrum of a therapy-refractory opportunist. Med. Mycol. 44, 295-327. doi: 10.1080/13693780600752507

Kaur, S., and Singh, S. (2014). Biofilm formation by Aspergillus fumigatus. Med. Mycol. 52, 2-9. doi: 10.3109/13693786.2013.819592

Kojic, E. M., and Darouiche, R. O. (2004). Candida infections of medical devices. Clin. Microbiol. Rev. 17, 255-267. doi: 10.1128/CMR.17.2.255-267.2004

Lamaris, G. A., Chamilos, G., Lewis, R. E., Safdar, A., Raad, I. I., and Kontoyiannis, D. P. (2006). Scedosporium infection in a tertiary care cancer center: a review of 25 cases from 1989-2006. Clin. Infect. Dis. 43, 1580-1584. doi: 10.1086/509579

Mello, T. P., Aor, A. C., Goncalves, D. S., Seabra, S. H., Branquinha, M. H., and Santos, A. L. (2016). Assessment of biofilm formation by Scedosporium apiospermum, S. aurantiacum, S. minutisporum and Lomentospora prolificans. Biofouling 32, 737-749. doi: 10.1080/08927014.2016.1192610

Mitchell, K. F., Zarnowski, R., and Andes, D. R. (2016). The extracellular matrix of fungal biofilms. Adv. Exp. Med. Biol. 931, 21-35. doi: 10.1007/5584_2016_6

Muller, F. M., Seidler, M., and Beauvais, A. (2011). Aspergillus fumigatus biofilms in the clinical setting. Med. Mycol. 49(Suppl. 1), S96-S100. doi: 10.3109/13693786. 2010.502190

Nett, J. E., Crawford, K., Marchillo, K., and Andes, D. R. (2010). Role of Fks1p and matrix glucan in Candida albicans biofilm resistance to an echinocandin, pyrimidine, and polyene. Antimicrob. Agents Chemother. 54, 3505-3508. doi: 10.1128/AAC.00227-10

Nunes, J. M., Bizerra, F. C., Ferreira, R. C., and Colombo, A. L. (2013). Molecular identification, antifungal susceptibility profile, and biofilm formation of clinical and environmental Rhodotorula species isolates. Antimicrob. Agents Chemother. 57, 382-389. doi: 10.1128/AAC.01647-12

Perez, R. E., Smith, M., McClendon, J., Kim, J., and Eugenio, N. (1988). Pseudallescheria boydii brain abscess, complication of an intravenous catheter. Am. J. Med. 84, 359-362. doi: 10.1016/0002-9343(88)90441-X
Pierce, C. G., Uppuluri, P., Tristan, A. R., Wormley, FL Jr, Mowat, E., Ramage, G., et al. (2008). A simple and reproducible 96-well plate-based method for the formation of fungal biofilms and its application to antifungal susceptibility testing. Nat. Protoc. 3, 1494-1500. doi: 10.1038/nport.2008.141

Rajendran, R., Mowat, E., McCulloch, E., Lappin, D. F., Jones, B., Lang, S., et al. (2011). Azole resistance of Aspergillus fumigatus biofilms is partly associated with efflux pump activity. Antimicrob. Agents Chemother. 55, 2092-2097. doi: 10.1128/AAC.01189-10

Rajendran, R., Sherry, L., Nile, C. J., Sherriff, A., Johnson, E. M., Hanson, M. F., et al. (2016). Biofilm formation is a risk factor for mortality in patients with Candida albicans bloodstream infection-Scotland, 2012-2013. Clin. Microbiol. Infect. 22, 87-93. doi: 10.1016/j.cmi.2015.09.018

Ramage, G., Rajendran, R., Gutierrez-Correa, M., Jones, B., and Williams, C. (2011). Aspergillus biofilms: clinical and industrial significance. FEMS Microbiol. Lett. 324, 89-97. doi: 10.1111/j.1574-6968.2011.02381.x

Reichhardt, C., Ferreira, J. A., Joubert, L. M., Clemons, K. V., Stevens, D. A., and Cegelski, L. (2015). Analysis of the Aspergillus fumigatus biofilm extracellular matrix by solid-state nuclear magnetic resonance spectroscopy. Eukaryot. Cell 14, 1064-1072. doi: 10.1128/EC.00050-15

Rougeron, A., Schuliar, G., Leto, J., Sitterlé, E., Landry, D., Bougnoux, M. E., et al. (2015). Human-impacted areas of France are environmental reservoirs of the Pseudallescheria boydii/Scedosporium apiospermum species complex. Environ. Microbiol. 17, 1039-1048. doi: 10.1111/1462-2920.12472

Schindelin, J., Arganda-Carreras, I., Frise, E., Kaynig, V., Longair, M., Pietzsch, T., et al. (2012). Fiji: an open-source platform for biological-image analysis. Nat. Methods 9, 676-682. doi: 10.1038/nmeth.2019

Seidler, M. J., Salvenmoser, S., and Muller, F. M. (2008). Aspergillus fumigatus forms biofilms with reduced antifungal drug susceptibility on bronchial epithelial cells. Antimicrob. Agents Chemother. 52, 4130-4136. doi: 10.1128/ AAC.00234-08

Sherry, L., Rajendran, R., Lappin, D. F., Borghi, E., Perdoni, F., Falleni, M., et al. (2014). Biofilms formed by Candida albicans bloodstream isolates display phenotypic and transcriptional heterogeneity that are associated with resistance and pathogenicity. BMC Microbiol. 14:182. doi: 10.1186/1471-2180-14-182

Summerbell, R. C., Krajden, S., and Kane, J. (1989). Potted plants in hospitals as reservoirs of pathogenic fungi. Mycopathologia 106, 13-22. doi: 10.1007/ BF00436921

Tadros, T. S., Workowski, K. A., Siegel, R. J., Hunter, S., and Schwartz, D. A. (1998). Pathology of hyalohyphomycosis caused by Scedosporium apiospermum (Pseudallescheria boydii): an emerging mycosis. Hum. Pathol. 29, 1266-1272. doi: 10.1016/S0046-8177(98)90255-6

Taj-Aldeen, S. J., Salah, H., Al-Hatmi, A. M., Hamed, M., Theelen, B., van Diepeningen, A. D., et al. (2016). In vitro resistance of clinical Fusarium species to amphotericin B and voriconazole using the EUCAST antifungal susceptibility method. Diagn. Microbiol. Infect. Dis. 85, 438-443. doi: 10.1016/j.diagmicrobio. 2016.05.006

Tammer, I., Tintelnot, K., Braun-Dullaeus, R. C., Mawrin, C., Scherlach, C., Schluter, D., et al. (2011). Infections due to Pseudallescheria/Scedosporium species in patients with advanced HIV disease-a diagnostic and therapeutic challenge. Int. J. Infect. Dis. 15, e422-e429. doi: 10.1016/j.ijid.2011.03.004

Tumbarello, M., Posteraro, B., Trecarichi, E. M., Fiori, B., Rossi, M., Porta, R., et al. (2007). Biofilm production by Candida species and inadequate antifungal therapy as predictors of mortality for patients with candidemia. J. Clin. Microbiol. 45, 1843-1850. doi: 10.1128/JCM.00131-07

Uppuluri, P., Chaturvedi, A. K., Srinivasan, A., Banerjee, M., Ramasubramaniam, A. K., Kohler, J. R., et al. (2010). Dispersion as an important step in the Candida albicans biofilm developmental cycle. PLoS Pathog. 6:e1000828. doi: 10.1371/ journal.ppat.1000828

Vediyappan, G., Rossignol, T., and d'Enfert, C. (2010). Interaction of Candida albicans biofilms with antifungals: transcriptional response and binding of antifungals to beta-glucans. Antimicrob. Agents Chemother. 54, 2096-2111. doi: 10.1128/AAC.01638-09

Vila, T. V., Chaturvedi, A. K., Rozental, S., and Lopez-Ribot, J. L. (2015). In vitro activity of Miltefosine against Candida albicans under Planktonic and biofilm growth conditions and in vivo efficacy in a murine model of oral candidiasis. Antimicrob. Agents Chemother. 59, 7611-7620. doi: 10.1128/AAC.01890-15

Vila, T. V., Ishida, K., de Souza, W., Prousis, K., Calogeropoulou, T., and Rozental, S. (2013). Effect of alkylphospholipids on Candida albicans 
biofilm formation and maturation. J. Antimicrob. Chemother. 68, 113-125. doi: $10.1093 / \mathrm{jac} / \mathrm{dks} 353$

Wilson, H. L., and Kennedy, K. J. (2013). Scedosporium apiospermum brain abscesses in an immunocompetent man with silicosis. Med. Mycol. Case Rep. 2, 75-78. doi: 10.1016/j.mmcr.2013. 02.006

Zouhair, R., Rougeron, A., Razafimandimby, B., Kobi, A., Bouchara, J. P., and Giraud, S. (2013). Distribution of the different species of the Pseudallescheria boydii/Scedosporium apiospermum complex in French patients with cystic fibrosis. Med. Mycol. 51, 603-613. doi: 10.3109/13693786.2013. 770606
Conflict of Interest Statement: The authors declare that the research was conducted in the absence of any commercial or financial relationships that could be construed as a potential conflict of interest.

Copyright $\odot 2017$ Rollin-Pinheiro, de Meirelles, Vila, Fonseca, Alves, Frases, Rozental and Barreto-Bergter. This is an open-access article distributed under the terms of the Creative Commons Attribution License (CC BY). The use, distribution or reproduction in other forums is permitted, provided the original author(s) or licensor are credited and that the original publication in this journal is cited, in accordance with accepted academic practice. No use, distribution or reproduction is permitted which does not comply with these terms. 